\title{
Combinatorial Hopf algebra of supercharacters of type $D$
}

\section{Carolina Benedettil|}

York University, Toronto

\begin{abstract}
We provide a Hopf algebra structure on the supercharacter theory for the unipotent upper triangular group of type $D$ over a finite field. Also, we make further comments with respect to types $B$ and $C$. Type $A$ was explored by M. Aguiar et. al (2010), thus this extended abstract is a contribution to understand combinatorially the supercharacter theory of the other classical Lie types.

Résumé. Nous construisons une structure d'algèbre de Hopf sur la thérie des supercharactères du groupe de matrices unipotentes triangulaires supéieures de type $D$. Nous donnons aussi quelques commentaires à l'égard des types $B$ et $C$. Le type $A$ a été explorée par M. Aguiar et al. (2010), donc ce résumé étendu est une contribution à la théorie combinatoire des supercharactères pour les autres types de Lie classiques.

Resumen. Dotamos con una estructura de álgebra de Hopf la teoría de supercaracteres del grupo de matrices unipotentes triangulares superiores de tipo $D$ sobre un cuerpo finito. Además, discutimos brevemente los tipos $B$ y $C$. El tipo $A$ fue explorado por M. Aguiar et al (2010), por lo tanto este resumen extendido es una contribución para entender combinatoriamente la teoría de supercaracteres de los otros tipos de Lie clásicos.
\end{abstract}

Keywords: Supercharacters, set partitions of type $D$, Hopf algebras, unipotent upper triangular groups.

\section{Introduction}

The problem of simultaneously reducing to canonical form two linear operators on a finite-dimensional space is a "wild" problem in representation theory. This problem contains all classification matrix problems given by quivers (see [BS03, Bon68]). In this sense, the classical representation theory for the type $A$ group $U_{n}(q)$ of unipotent $n \times n$ upper triangular matrices over a finite field is known to be wild. This makes, in some sense, hopeless any attempt to study the representation theory of the group $U_{n}(q)$. In his Ph.D. thesis C. Andre started to develop a theory that approximates the "wildness" of $U_{n}(q)$. Roughly speaking, by using certain linear combinations of irreducible characters and lumping together conjugacy classes under certain conditions, the resulting theory behave very nicely (see [And95, Thi10]). This gave rise to the concept of "supercharacter theory". Later on, P. Diaconis and I. M. Isaacs extended this concept to arbitrary algebra groups (see [DI08]). Supercharacter theory of the group $U_{n}(q)$ has a rich combinatorics which allows to connect this beautiful theory with classical combinatorial objects. As a matter of

${ }^{\dagger}$ NSERC and CRC (N. Bergeron's ). Partially supported by Beca Mazda para el Arte y la Ciencia.

1365-8050 @ 2012 Discrete Mathematics and Theoretical Computer Science (DMTCS), Nancy, France 
fact, in $\left[\mathrm{AAB}^{+} 11\right]$ a Hopf algebra structure is provided on the vector space $\mathbf{S C}$ of superclass characteristic functions over $U_{n}(q)$, for $n \geq 0$. Moreover, when $q=2$ this Hopf algebra is a realization of a well-known combinatorial Hopf algebra, namely, the Hopf algebra of symmetric functions in noncommuting variables (see [Sag01, BZ09]).

The reader familiar with the classical representation theory of the symmetric group $S_{n}$ will notice how this resembles the relationship between symmetric functions and the character theory of $S_{n}$. Also, supercharacters of $U_{n}(2)$ are indexed by set partitions of the set $[n]=\{1,2, \ldots, n\}$ and by labelled set partitions for general $q$.

In this paper, we study combinatorially the supercharacter theory corresponding to the other classical Lie types $B, C$ and $D$, making emphasis on the latter. These groups fail to be algebra groups unlike type $A$, however, we can regard them as subgroups of the convenient group of type $A$ and restrict the supercharacter theory of type $A$ to the respective subgroup. Nevertheless, we will see that the product defined on type $D$, in particular, differs from the one defined for type $A$ in $\left[\mathrm{AAB}^{+} 11\right]$. The paper is organized as follows. In Section 2 we provide the reader with the basic definitions concerning supercharacters. In Section 3. we give a combinatorial interpretation for the supercharacter theory of the group $U_{2 n}^{D}(q)$ of even orthogonal unipotent upper triangular matrices with coefficients on the field $\mathbb{F}_{q}$ of characteristic $\geq 3$. This combinatorial interpretation is using labelled $D_{2 n}$-partitions of the set $[ \pm n]:=\{1, \ldots, n,-n, \ldots,-1\}$. More specifically, we use these partitions to index orbit representatives for superclasses and supercharacters of the group $U_{2 n}^{D}(q)$. Then, we define the analog of $\mathbf{S C}$ for type $D$ as follows:

$$
\begin{aligned}
\mathbf{S C}^{D} & =\oplus_{n \geq 0} \mathbf{S C}_{n}^{D} \\
& =\oplus_{n \geq 0} \operatorname{span}_{\mathbb{C}}\left\{\kappa_{\lambda}: \lambda \in D_{2 n}(q)\right\}
\end{aligned}
$$

where $\kappa_{\lambda}$ denotes the superclass characteristic function indexed by the labelled $D_{2 n}$-partition $\lambda$. Using a change of basis, we prove that the space $\mathbf{S C}^{D}$ is endowed with a Hopf algebra structure.

Finally, we discuss briefly the supercharacter theory for types $B$ and $C$, concluding the study of the classical Lie types. Also we make an important remark concerning the Hopf monoid structure that $\mathbf{S C}^{D}$ carries, following the results in [ABT11].

\section{Preliminaries}

We start defining the supercharacter theory for a finite group $G$.

Definition 2.1 A supercharacter theory for $G$ consists of:

- A partition $\mathcal{K}$ of $G$

- A set $\mathcal{X}$ of characters of $G$

such that the following holds:

1. $|\mathcal{K}|=|\mathcal{X}|$

2. every irreducible character of $G$ is a constituent of a unique $\chi \in \mathcal{X}$

3. the characters on $\chi$ are constant on members of $\mathcal{K}$. 
The elements in $\mathcal{X}$ can be thought of as scalar multiples of linear combinations of the form $\sum_{\psi \in X} \psi(1) \psi$ where $X$ is a subset of irreducible characters of $G$.

Remark: Definition 2.1 is equivalent to say that $\operatorname{span}_{\mathbb{C}}\left\{\sum_{g \in K} g: K \in \mathcal{K}\right\}$ is a subalgebra of $Z(\mathbb{C} G)$ with unit 1 . Given such partition $\mathcal{K}$, there exists $\mathcal{X}$ with the desired properties.

Examples 2.2 - Every group is endowed with the trivial supercharacter theory where the set of superclasses $\mathcal{K}$ consists of the usual conjugacy classes and the set of supercharacters $\chi$ is formed by the irreducible representations of $G$.

- Similarly, the coarsest supercharacter theory of $G$ is such that $\mathcal{K}=\{\{1\}, G-\{1\}\}$ and $\chi=$ $\left\{1, \rho_{G}-1\right\}$, where $\rho_{G}$ is the regular representation.

As mentioned in the introduction, this paper explores the supercharacter theory of the classical group of $2 n \times 2 n$ unipotent upper triangular matrices of type $D$, which we denote by $U_{2 n}^{D}(q)$. We regard $U_{2 n}^{D}(q)$ as a subgroup of the group $U_{2 n}(q)$ of $2 n \times 2 n$ unipotent upper triangular matrices, which is an algebra group as defined below.

Definition 2.3 Let $J$ be a finite dimensional nilpotent $\mathbb{F}$-algebra and let $G$ denote the set consisting of formal objects of the form $1+a$ where $a \in J$. Then $G=1+F$ is a group, where the multiplication is given by $(1+a)(1+b)=1+a+b+a b$. The group $G$ is the algebra group based on $J$.

As an example, denote by $\mathfrak{u}_{n}$ the algebra of nilpotent upper triangular matrices associated to the group $U_{n}(q)$. Then we see that $U_{n}(q)=I+\mathfrak{u}_{n}$, thus $U_{n}(q)$ is an algebra group.

The supercharacter theory for the group $U_{n}(q)$ has a very nice combinatorial interpretation. Its superclasses are indexed by labelled set partitions of type $A$ as well as its supercharacters (see [AAB $\left.{ }^{+} 11\right]$ ). In analogy with type $A$, in the next section we describe the supercharacter theory for the group $U_{2 n}^{D}(q)$ using labelled $D_{2 n}$-partitions, though as mentioned in the introduction, $U_{2 n}^{D}(q)$ is not an algebra group.

\section{Supercharacter theory of type $D$}

From now on, $\mathbb{F}_{q}$ will denote a field of characteristic $p \geq 3$ and order $q=p^{r}$ for some integer $r \geq 1$. The group $U_{2 n}^{D}(q)$ corresponds to even orthogonal unipotent upper triangular matrices with coefficients in $\mathbb{F}_{q}$ and can be described as follows (see [Car72]):

$$
U_{2 n}^{D}(q)=\left\{\left(\begin{array}{cc}
P & P Q \\
0 & J P^{-t} J
\end{array}\right): P \in U_{n}(q), Q \in M_{n}(q), J Q^{t} J=-Q\right\} .
$$

where $M_{n}(q)$ is the set of $n \times n$ matrices over $\mathbb{F}_{q}$ and $J$ is the $n \times n$ matrix with ones in the antidiagonal and zeros elsewhere.

We will drop the subindex $2 n$ from the notation, but keeping in mind the size of the matrices. In order to describe the superclasses for this type, we make use of the nilpotent algebra $\mathfrak{u}^{D}(q)$ associated to the group $U^{D}(q)$. Denote by $\mathfrak{u}^{D}(q)$ the algebra given by

$$
\mathfrak{u}^{D}(q)=\left\{\left(\begin{array}{cc}
R & Q \\
0 & -J R^{t} J
\end{array}\right): R \in U_{n}(q)-I_{n}, Q \in M_{n}(q), J Q^{t} J=-Q\right\} .
$$


with $M_{n}(q)$ and $J$ as before. We will make use of the total order

$$
1 \prec \cdots \prec n \prec-n \prec \cdots \prec-1
$$

to index the columns and rows of matrices in $U^{D}(q)$ and in $\mathfrak{u}^{D}(q)$, from left to right and top to bottom.

A vector space basis for $\mathfrak{u}^{D}(q)$ over $\mathbb{F}_{q}$ is given by the matrices $\left\{y_{\alpha}\right\}_{\alpha}$ where $\alpha$ runs over the set of positive roots $\Phi^{+}$of type $D$, given by

$$
\Phi^{+}=\left\{e_{i} \pm e_{j}: 1 \leq i<j \leq n\right\}
$$

and $y_{\alpha}$ denotes the matrix

$$
y_{\alpha}= \begin{cases}e_{i, j}-e_{-j,-i} & \text { if } \alpha=e_{i}-e_{j} \\ e_{i,-j}-e_{j,-i} & \text { if } \alpha=e_{i}+e_{j}\end{cases}
$$

where $e_{i, j} \in \mathfrak{u}^{D}(q)$ has 1 in position $i, j$ and zeros elsewhere. Now define the support of $y_{\alpha}$ by

$$
\operatorname{supp}\left(y_{\alpha}\right)= \begin{cases}(i, j),(-j,-i) & \text { if } \alpha=e_{i}-e_{j} \\ (i,-j),(j,-i) & \text { if } \alpha=e_{i}+e_{j}\end{cases}
$$

Notice that this definition can be extended linearly to the whole $\mathfrak{u}^{D}(q)$.

Combinatorially, linear combinations of the matrices $y_{\alpha}$ with at most one nonzero entry in every row and column can be seen as labelled $D_{2 n}$-partitions or simply $D_{2 n}(q)$-partitions. These partitions are determined by their set of arcs labelled by nonzero elements of $\mathbb{F}_{q}$ as follows:

Definition 3.1 Let $a, b \in \mathbb{F}_{q}^{*}$. A $D_{2 n}(q)$-partition $\lambda$ of $[ \pm n]$ is given by a set of labelled arcs in $[ \pm n]$ such that for $j \neq-i$ :

(a). If $i \stackrel{a}{\sim} j \in \lambda$ then $-j \stackrel{-a}{\sim}-i \in \lambda$

(b). If $i \stackrel{a}{\sim} j \in \lambda$ and $i \prec k \prec j$ then $i \stackrel{b}{\circ} k, k \stackrel{b}{5} j \notin \lambda$.

We say that $\lambda \in D_{2 n}(q)$.

For $\lambda \in D_{2 n}(q)$, the corresponding matrix $y_{\lambda} \in \mathfrak{u}^{D}(q)$ is then given by

$$
y_{\lambda}=\sum_{i{ }_{i}^{a} j \in \lambda} a e_{i, j} .
$$

This allows us to go back and forth (in a unique way) between set partitions in $D_{2 n}(q)$ and matrices in $\mathfrak{u}^{D}(q)$ with at most one non zero element in every row and column.

Every $\lambda \in D_{2 n}(q)$ can be written uniquely as $\lambda=\lambda^{+} \cup \lambda^{-}$, where:

- $\lambda^{+} \cap \lambda^{-}=\emptyset$

- $i \stackrel{a}{\sim} j \in \lambda^{+}$if and only if $-j \stackrel{-a}{\stackrel{a}{-}}-i \in \lambda^{-}$where $i>0$ and $i<|j|$

In view of this, $\lambda$ is completely determined by $\lambda^{+}$(or $\lambda^{-}$). Also, every arc $i \stackrel{a}{\sim} j \in \lambda^{+}$can be represented by the triple $\{(i, j, a)\}$. In this case, the triple $\{(-j,-i,-a)\} \in \lambda^{-}$. 


\subsection{Superclasses and supercharacters}

In this section we describe combinatorially the superclasses and supercharacters of $U_{2 n}^{D}(q)$ using $D_{2 n}(q)$ partitions and keeping in mind that $U_{2 n}^{D}(q)$ is a subgroup of $U_{2 n}(q)$. Using algebraic varieties, André and Neto proved that supercharacters and superclasses of the group $U_{2 n}^{D}(q)$ are indexed by matrices in $\mathfrak{u}^{D}(q)$ with at most nonzero element in every row and column (see [AN09b]). Thus, they can be indexed using $D_{2 n}(q)$-partitions as well.

The group $U_{2 n}(q)$ acts on its nilpotent algebra $\mathfrak{u}_{2 n}(q)$ by left and right multiplication. It can be shown that when adding the identity $I_{2 n}$ to each one of these orbits we get the superclasses of $U_{2 n}(q)$ (see [DI08]).

Let $\lambda \in D_{2 n}(q)$ and let $y_{\lambda}$ as in 3.1 . Since $\mathfrak{u}^{D}(q) \subset \mathfrak{u}_{2 n}(q)$ we can consider the orbit

$$
V_{\lambda}=U_{2 n}(q) y_{\lambda} U_{2 n}(q) \in \mathfrak{u}_{2 n}(q) .
$$

Notice that $V_{\lambda}$ is not necessarily in $\mathfrak{u}^{D}(q)$. However, since $V_{\lambda}+I_{2 n}$ is a superclass in $U_{2 n}(q)$ and $U_{2 n}^{D}(q)$ is a subgroup of $U_{2 n}(q)$, we define the superclass in $U_{2 n}^{D}(q)$ associated to $\lambda$ as $K_{\lambda}:=U^{D}(q) \cap\left(V_{\lambda}+I_{2 n}\right)$.

As mentioned in the introduction $U_{2 n}^{D}(q)$ is not an algebra group, i.e., $U_{2 n}^{D}(q) \neq I_{2 n}+\mathfrak{u}_{2 n}^{D}(q)$. Yet there is a bijective correspondence between $U_{2 n}^{D}(q)$ and $\mathfrak{u}^{D}(q)$. This bijection is provided by the following lemma.

Lemma 3.2 ( [AN09b], Lemma 2.3) Let $\lambda$ be a $D_{2 n}$-partition. Put $x$ and $y$ as

$$
x=\left(\begin{array}{c|c}
P & P Q \\
\hline 0 & J P^{-t} J
\end{array}\right) \in U^{D}(q) \quad \text { and } \quad y=\left(\begin{array}{c|c}
P-1 & Q \\
\hline 0 & -J(P-1)^{t} J
\end{array}\right) \in \mathfrak{u}^{D}(q) .
$$

Then $x \in K_{\lambda}$ if and only if $y \in V_{\lambda}$.

To illustrate this lemma, let us consider the following example:

Example 3.3 Let $n=5$ and let $\lambda$ be the $D_{2 n}(q)$-partition given by

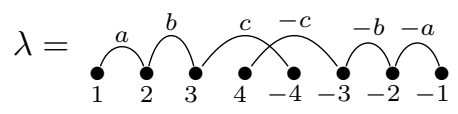

A natural representative for the orbit $V_{\lambda}$ is given by the corresponding $y_{\lambda}$. In this example we have

$y_{\lambda}=\left(\begin{array}{cccc|cccc}0 & a & 0 & 0 & 0 & 0 & 0 & 0 \\ 0 & 0 & b & 0 & 0 & 0 & 0 & 0 \\ 0 & 0 & 0 & 0 & c & 0 & 0 & 0 \\ 0 & 0 & 0 & 0 & 0 & -c & 0 & 0 \\ 0 & 0 & 0 & 0 & 0 & 0 & 0 & 0 \\ 0 & 0 & 0 & 0 & 0 & 0 & -b & 0 \\ 0 & 0 & 0 & 0 & 0 & 0 & 0 & -a \\ 0 & 0 & 0 & 0 & 0 & 0 & 0 & 0\end{array}\right) \quad$ and $\quad x_{\lambda}=\left(\begin{array}{cccc|cccc}1 & a & 0 & 0 & 0 & 0 & 0 & 0 \\ 0 & 1 & b & 0 & 0 & 0 & 0 & 0 \\ 0 & 0 & 1 & 0 & c & b c & 0 & 0 \\ 0 & 0 & 0 & 1 & 0 & -c & 0 & 0 \\ 0 & 0 & 0 & 0 & 1 & 0 & 0 & 0 \\ 0 & 0 & 0 & 0 & 0 & 1 & -b & 0 \\ 0 & 0 & 0 & 0 & 0 & 0 & 1 & -a \\ 0 & 0 & 0 & 0 & 0 & 0 & 0 & 1\end{array}\right)$

where $x_{\lambda}$ is the matrix in $K_{\lambda}$ given by the lemma 3.2 
Notice that $x_{\lambda} \neq y_{\lambda}+I_{10}$, but the bijection provided by the lemma makes it easier to get a representative $x_{\lambda}$ for the superclass $K_{\lambda}$ by computing first $y_{\lambda} \in V_{\lambda}$. This is very useful especially when the partition $\lambda$ is more complicated.

Let $\theta: \mathbb{F}_{q} \rightarrow \mathbb{C}^{*}$ be any nontrivial group homomorphism. This homomorphism will be kept fixed from now on. As before, $\mathfrak{u}$ denotes the nilpotent upper triangular matrices of type $A$. Let $\mathfrak{u}^{*}$ be the vector space dual to $\mathfrak{u}$ and let $e_{i, j}^{*}$ be the element in $\mathfrak{u}^{*}$ dual to $e_{i, j} \in \mathfrak{u}$ so that $e_{i, j}^{*}\left(e_{i, j}\right)=\delta_{i, j}$. Supercharacters of the group $U_{2 n}(q)$ are given by the double action of the group $U_{2 n}(q)$ over the vector space $\mathfrak{u}^{*}$. This double action is given by

$$
(x \lambda y)(a)=\lambda\left(x^{-1} a y^{-1}\right)
$$

where $\lambda \in \mathfrak{u}^{*}, x, y \in U_{2 n}(q)$ and $a \in \mathfrak{u}$. Let $\tilde{\chi}^{\lambda}$ be the supercharacter of $U_{2 n}(q)$ indexed by $\lambda \in D_{2 n}(q)$. Since $U_{2 n}(q)$ contains $U_{2 n}^{D}(q)$ as a subgroup, we will denote by $\chi^{\lambda}$ the restriction of $\tilde{\chi}^{\lambda}$ to $U_{2 n}^{D}(q)$. Then $\chi^{\lambda}$ is the supercharacter in $U_{2 n}^{D}(q)$ indexed by $\lambda$.

The following theorem is a combinatorial way of defining supercharacters on superclasses:

Theorem 3.4 ( [AN09a], Theorem 5.3) Let $\lambda$ be a $D_{2 n}(q)$-partition and let $x_{\mu}$ be the superclass associated to the $D_{2 n}(q)$-partition $\mu$. Then

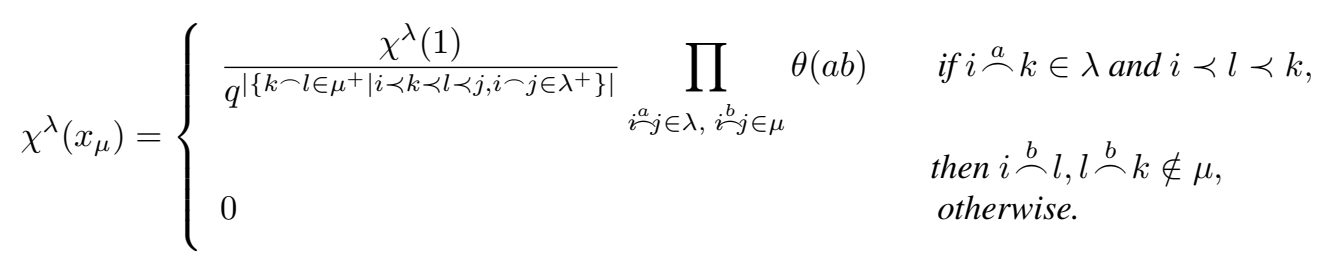

A few remarks are worth to mention about some the combinatorial properties of supercharacters. For an algebraic proof see [AN09b]:

$$
\begin{aligned}
& \text { - } \chi^{\lambda}(1)=\left\{\begin{array}{ll}
q^{j-i-1} & \text { if } j \preceq n \\
q^{2 n-i-j} & \text { otherwise }
\end{array} \text { when } \lambda^{+}=\{(i, j, a)\}\right. \text { is a single arc. } \\
& \text { - } \chi^{\lambda}=\prod_{\lambda_{i j} \in \lambda} \chi^{\lambda_{i j}} \quad \text { where } \lambda_{i j}=\{i \stackrel{a}{\sim} j,-j \stackrel{-a}{\sim}-i\} \in \lambda \text {. Thus, } \chi^{\lambda} \text { is one dimensional if and only if } \\
& j=i+1 \text { for every } \lambda_{i j} \text {. }
\end{aligned}
$$

\subsection{Product and coproduct}

Let $\mathbf{S C}_{n}^{D}$ be the vector space of superclass functions over the group $U_{2 n}^{D}(q)$. Now that we know how superclasses and supercharacters look like as matrices and as partitions, we will define a product and a coproduct on the graded vector space $\mathbf{S C}^{D}=\bigcup_{n \geq 1} \mathbf{S C}_{n}^{D}$. As proved in [AN09a, Theorem 4.1], the supercharacters of $U_{2 n}^{D}(q)$ form a basis for $\mathbf{S C}_{n}^{D}$.

In order to give a nice combinatorial description of the operations we will define, we use the basis of $\mathbf{S C}^{\mathbf{D}}$ given by the superclass characteristic functions $\kappa_{\lambda}$, i. e., 


$$
\mathbf{S C}^{\mathbf{D}}=\operatorname{span}_{\mathbb{C}}\left\{\kappa_{\lambda}: \lambda \in D_{2 n}(q), n \geq 1\right\}
$$

where

$$
\kappa_{\lambda}\left(x_{\mu}\right)= \begin{cases}1 & \text { if } x_{\mu} \text { is in the same superclass of } x_{\lambda} \\ 0 & \text { otherwise }\end{cases}
$$

Let $P^{-J}$ denote the matrix multiplication given by $J P^{-t} J$ for $P \in U_{2 n}(q)$. The subgroup $U_{2 k}^{D}(q) \times$ $U_{2(n-k)}^{D}(q)$ can be embedded into $U_{2 n}^{D}(q)$ by

$$
U_{2 k}^{D} \times U_{2(n-k)}^{D} \ni\left(\begin{array}{cc}
P_{1} & P_{1} Q_{1} \\
0 & P_{1}^{-J}
\end{array}\right) \times\left(\begin{array}{cc}
P_{2} & P_{2} Q_{2} \\
0 & P_{2}^{-J}
\end{array}\right) \hookrightarrow\left(\begin{array}{cc|cc}
P_{1} & 0 & 0 & P_{1} Q_{1} \\
0 & P_{2} & P_{2} Q_{2} & 0 \\
\hline 0 & 0 & P_{2}^{-J} & 0 \\
0 & 0 & 0 & P_{1}^{-J}
\end{array}\right)
$$

On the other hand, let $\tau: U_{2 n}^{D}(q) \rightarrow U_{2 k}^{D}(q) \times U_{2(n-k)}^{D}(q)$ be the surjective homomorphism given by

$$
\left(\begin{array}{c|c}
P & P Q \\
\hline 0 & J P^{-t} J
\end{array}\right) \mapsto\left(\begin{array}{cc|cc}
P_{1} & 0 & 0 & P_{1} Q_{1} \\
0 & P_{2} & P_{2} Q_{2} & 0 \\
\hline 0 & 0 & P_{2}^{-J} & 0 \\
0 & 0 & 0 & P_{1}^{-J}
\end{array}\right)
$$

where $P=\left(\begin{array}{cc}P_{1} & P_{3} \\ 0 & P_{2}\end{array}\right)$ and $Q=\left(\begin{array}{cc}R & Q_{1} \\ Q_{2} & S\end{array}\right)$ are decompositions of the matrices $P, Q$. Notice that the matrices $Q_{1}, Q_{2}$ satisfy the same property as the matrix $Q$, namely, $J Q^{t} J=-Q$. Here we are abusing notation by putting 0 in the matrices, but they should be understood as zero blocks of the correct size.

To simplify notation, we will put $U^{D}=U^{D}(q)$ but the reader should keep in mind that the ground field is $\mathbb{F}_{q}$. In order to give an explicit formula for the product on $\mathbf{S C}^{D}$, we need the inflation map on supercharacters, which is given by

$$
\operatorname{Inf}_{U_{2 k}^{D} \times U_{2(n-k)}^{D}}^{U^{D}}(\chi \times \psi)\left(u_{\nu}\right)=(\chi \times \psi)(\tau(x))
$$

where $x$ is a superclass in $U_{2 n}^{D}$ and $\chi \times \psi \in \mathbf{S C}_{2 k}^{D} \times \mathbf{S C}_{2(n-k)}^{D}$. Thus, defining the product of supercharacters by

$$
\chi \cdot \psi=\operatorname{Inf}_{U_{2 k}^{D} \times U_{2(n-k)}^{D}}^{U^{D}}(\chi \times \psi)
$$

allow us to get a nice combinatorial formula for the product of superclass characteristic functions.

Proposition 3.5 For $\lambda, \mu$ labelled D-partitions of $[ \pm k],[ \pm(n-k)]$, respectively, we have

$$
\kappa_{\lambda} \cdot \kappa_{\mu}=\sum_{\nu=\lambda \sqcup \gamma \sqcup(k+\mu)} \kappa_{\nu}
$$

where 
- $i \stackrel{a}{\sim} j \in \gamma \Rightarrow i \preceq k \prec j$ or

- $i \stackrel{a}{\sim}-j \in \gamma \Rightarrow-j \prec-k \preceq-i$

for $1 \leq i, j \leq n$.

In our notation, for $1 \leq i, j \leq n-k$

$$
k+\mu= \begin{cases}(k+i) \stackrel{a}{a}(k+j) & \text { if } i \stackrel{a}{a} j \in \mu \\ (k+i) \stackrel{a}{a}(-k-j) & \text { if } i \stackrel{a}{\circ}-j \in \mu\end{cases}
$$

It is straightforward to check that $\left(\kappa_{\lambda} \cdot \kappa_{\mu}\right) \cdot \kappa_{\nu}=\kappa_{\lambda} \cdot\left(\kappa_{\mu} \cdot \kappa_{\nu}\right)$ for any $\lambda, \mu, \nu$. This makes the $\cdot$ operation associative, and the unit is given by $\kappa_{\emptyset}$. Also notice that this product is noncommutative.

Before illustrating this proposition with an example, as mentioned in the introduction, this product differs from the one defined for $\mathbf{S C}$ in $\left[\mathrm{AAB}^{+} 11\right]$. The difference is that here we do not concatenate $\lambda$ and $\mu$. Instead, we put $\mu$ in between $\lambda^{+}$and $\lambda^{-}$. This resembles the product defined in [AL11, Section 3.5] for the Hopf monoid Pal of palindromic set compositions.

Example 3.6 Denote $-i$ by $\bar{i}$, then

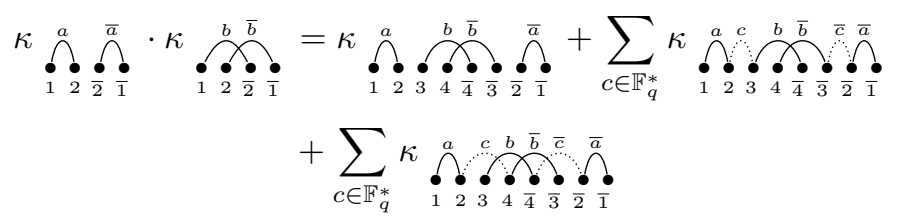

With the definition of product on $\mathbf{S C}^{D}$ given, we want to define a coproduct. First we define the standardization map st $\mathbf{s t}_{J}$ on ordered $D$-set partitions $J=\left\{J_{1}|\ldots| J_{r}\right\}$ of $[ \pm n]$. These are partitions such that $J_{i}=-J_{i}$ and $\cup J_{i}=[ \pm n]$ for all $i$. In fact, for our purposes it is enough to define $\mathbf{s t}_{J}$ for ordered $D$-set partitions $J=\left(J_{1} \mid J_{2}\right)$ of size at most 2 . To simplify notation, if $J=\left( \pm A \mid \pm A^{c}\right)=(13 \overline{1} \overline{3} \mid 24 \overline{2} \overline{4})$, we simply write $J=(13 \mid 24)$ so that $A=\{1,3\}$. In this case, $\pm A= \pm\{1,3\}$ so that $[ \pm|A|]=[ \pm 2]$.

Let $J=\left(A \mid A^{c}\right)$ be an ordered $D$-set partition of $[ \pm n]$ and let

$$
S_{J}(q)=\left\{\lambda \in D_{2 n}(q): i \stackrel{a}{\sim} j \in \lambda \text { implies } i, j \text { are in the same part of } J\right\}
$$

and define the bijection

$$
\text { st }_{J}: S_{J}(q) \rightarrow S_{[ \pm|A|]}(q) \times S_{\left[ \pm\left|A^{c}\right|\right]}(q)
$$

that relabels the indices of partitions in $S_{J}(q)$ according to the unique order-preserving map

$$
\mathrm{st}_{A}: \pm A \rightarrow[ \pm|A|]
$$

where $A$ is a part of $J$.

As an example, let $J=\{134 \mid 25\}$ and let $\lambda$ be given by

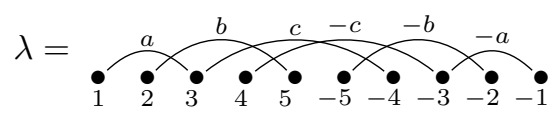


then

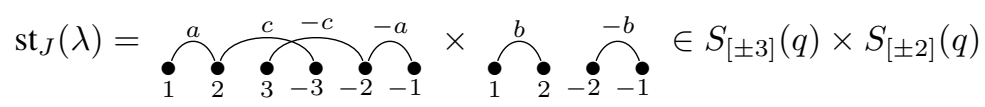

Definition 3.7 Let $J$ be an ordered D-set partition of $[ \pm n]$. The subgroup $U_{J}^{D}$ of $U^{D}=U_{2 n}^{D}(q)$ is given by

$$
U_{J}^{D}=\left\{x \in U^{D}: x_{i j} \neq 0 \text { implies } i, j \text { are in the same part of } J\right\}
$$

The map in 3.6 can be extended to produce an isomorphism st $\operatorname{st}_{J}: U_{J}^{D} \rightarrow U_{2|A|}^{D}(q) \times U_{2\left|A^{c}\right|}^{D}(q)$ by reordering the rows and columns as in 3.7 .

The restriction map is then given by

$$
\operatorname{Res}_{\text {st }_{J}\left(U_{J}^{D}\right)}^{U^{D}}: \mathbf{S C}_{2 n}^{D}(q) \rightarrow \mathbf{S C}_{2|A|}^{D}(q) \otimes \mathbf{S C}_{2\left|A^{c}\right|}^{D}(q)
$$

where $\operatorname{Res}_{\mathrm{st}_{J}\left(U_{J}^{D}\right)}^{U^{D}}(\chi)(u)=\chi\left(\mathrm{st}_{J}^{-1}(u)\right)$ for $u \in U_{2|A|}^{D}(q) \times U_{2\left|A^{c}\right|}^{D}(q)$.

Now, define the coproduct on supercharacters as

$$
\Delta(\chi)=\sum_{\substack{J=\left(A \mid A^{c}\right) \\ A \subseteq[ \pm n], A=-A}} \operatorname{Res}_{\mathrm{st}_{J}\left(U_{J}^{D}\right)}^{U^{D}}(\chi)
$$

then we have the following result dual to proposition 3.5

Proposition 3.8 Let $\lambda$ be a $D_{2 n}(q)$-partition. Then

$$
\Delta\left(\kappa_{\lambda}\right)=\sum_{\lambda=\mu \sqcup \nu} \kappa_{s t_{A}(\mu)} \otimes \kappa_{s t_{A} c}(\nu)
$$

summing over $\mu, \nu$ such that $\left.\lambda\right|_{A}=\mu,\left.\lambda\right|_{A^{c}}=\nu$ for $A \subseteq[ \pm n]$ and $A=-A$.

Example 3.9 Let $\lambda \in D_{12}(q)$ given by

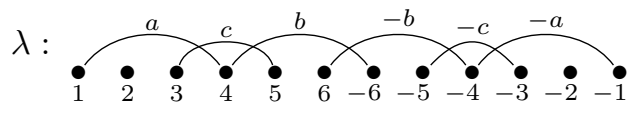

Then,

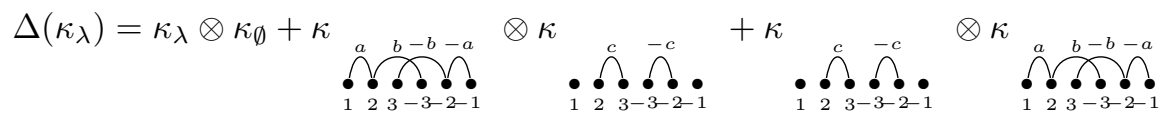

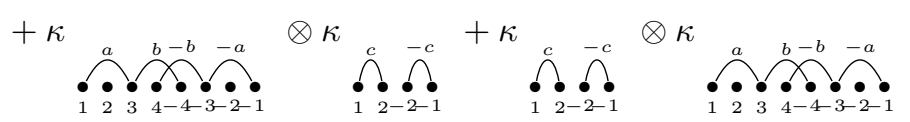

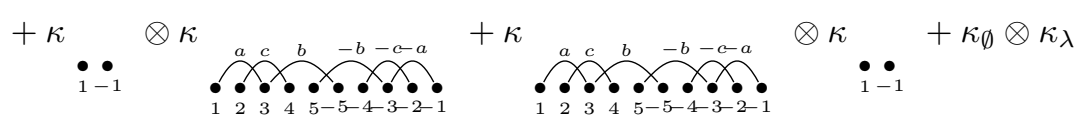


As with the product, it is not hard to see that the coproduct is associative.

We would like to show that the product and coproduct provide $\mathbf{S C}^{\mathbf{D}}$ with a bialgebra structure. For that purpose we would need the equality $\Delta\left(\kappa_{\mu} \cdot \kappa_{\nu}\right)=\Delta\left(\kappa_{\mu}\right) \cdot \Delta\left(\kappa_{\nu}\right)$ to hold. This equality will be proved using the P-basis whose definition we give below.

Definition 3.10 Let $\lambda$, $\mu$ be $D_{2 n}(q)$-partitions. We say that $\lambda \leq \mu$ if $\mathcal{A}(\lambda) \subseteq \mathcal{A}(\mu)$ where $\mathcal{A}(\lambda)$ denotes the set of $\operatorname{arcs}$ in $\lambda$.

Given $\lambda$, we denote by $P_{\lambda}$ the superclass function defined as

$$
P_{\lambda}:=\sum_{\mu \geq \lambda} \kappa_{\lambda}
$$

From here, we see that $\left\{P_{\lambda}\right\}_{\lambda \in D_{2 n}(q)}$ forms a basis for $\mathbf{S C}^{D}$ as $n \geq 0$. This basis is called $P$-basis.

Proposition 3.11 The P-basis multiplies and comultiplies as follows:

(a) For $\mu, \nu$ labelled D-partitions of $[ \pm k],[ \pm(n-k)]$, respectively, we have

$$
P_{\mu} \cdot P_{\nu}=P_{\mu \sqcup(k+\nu)}
$$

(b) For $\lambda \in D_{2 n}(q)$ we have

$$
\Delta\left(P_{\lambda}\right)=\sum_{\lambda=\mu \sqcup \nu} P_{s t_{A}(\mu)} \otimes P_{s t_{A c}(\nu)}
$$

summing over $\mu, \nu$ such that $\left.\lambda\right|_{A}=\mu,\left.\lambda\right|_{A^{c}}=\nu$ for $A \subseteq[ \pm n]$ and $A=-A$.

To conclude this chapter, we put all the pieces together to prove our main result.

Theorem 3.12 The product and coproduct given in proposition 3.11 provides the space $\boldsymbol{S C}^{D}$ with a Hopf algebra structure.

Proof: We prove only the compatibility relation between the product and the coproduct. Let $\lambda \in$ $D_{2 k}(q), \mu \in D_{2(n-k)}(q)$, then

$$
\begin{aligned}
\Delta\left(P_{\lambda}\right) \cdot \Delta\left(P_{\mu}\right)= & \left.\sum_{\substack{\lambda=\tau_{1} \sqcup \sigma_{1} \\
B \subseteq[ \pm], B=-B}} P_{\mathrm{st}_{B}\left(\tau_{1}\right)} \otimes P_{\mathrm{st}_{B}\left(\sigma_{1}\right)}\right)\left(\sum_{\substack{\mu=\tau_{2} \sqcup \sigma_{2} \\
C \subseteq[ \pm(n-k)], C=-C}} P_{\mathrm{st}_{C}\left(\tau_{2}\right)} \otimes P_{\mathrm{st}_{C^{c}}\left(\sigma_{2}\right)}\right) \\
= & \sum_{\substack{\lambda=\tau_{1} \sqcup \sigma_{1} \\
B=-B=\tau_{2} \sqcup \sigma_{2} \\
C=-C}} P_{\mathrm{st}_{B}\left(\tau_{1}\right) \sqcup \mathrm{st}_{C}\left(\tau_{2}\right)} \otimes P_{\mathrm{st}_{B^{c}}\left(\sigma_{1}\right) \sqcup \mathrm{st}_{C^{c}}\left(\sigma_{2}\right)} \\
= & \sum_{\substack{\lambda \sqcup \mu=\tau \sqcup \sigma \\
A \subseteq[ \pm n], A=-A}} P_{\mathrm{st}_{A}(\tau)} \otimes P_{\mathrm{st}_{A^{c}}(\sigma)} \text { where } \tau=\tau_{1} \sqcup \tau_{2}, \sigma=\sigma_{1} \sqcup \sigma_{2} \\
= & \Delta\left(P_{\lambda \sqcup \mu}\right)=\Delta\left(P_{\lambda} \cdot P_{\mu}\right)
\end{aligned}
$$




\section{Final comments}

We finish with a brief description of the supercharacter theory for types $B$ and $C$. Supercharacters and superclasses for these types are also indexed by labelled partitions of the corresponding type. The unipotent upper triangular matrices of type $B$ is the group of $m \times m$ orthogonal matrices where $m=2 n+1$ for some $n \in \mathbb{Z}_{\geq 0}$. We define $B_{m}(q)$-partitions as labelled partitions on the set $\{1, \ldots, n, 0,-n, \ldots,-1\}$ satisfying the same properties as $D_{2 n}(q)$-partitions with the additional property that we allow arcs of the form $i \stackrel{a}{\sim} 0$ together with $0 \stackrel{-a}{\sim}-i$.

Unfortunately, any attempt to construct a product on $\mathbf{S C}^{B}$ fails since dealing with odd-size matrices make impossible an embedding from $\mathbf{S C}_{2 k+1}^{B} \times \mathbf{S} \mathbf{C}_{2 l+1}^{B}$ to $\mathbf{S C}_{2(k+l)+1}^{B}$ in the sense of 3.3 . However, we have a structure of $\mathbf{S C}^{D}$-module on $\mathbf{S C}^{B}$, since it is clear that $\mathbf{S C}_{2 k}^{D} \times \mathbf{S} \mathbf{C}_{2 l+1}^{B}$ embeds into $\mathbf{S C}_{2(k+l)+1}^{B} \in \mathbf{S} \mathbf{C}^{B}$.

For unipotent upper triangular matrices of type $C$ the situation is better. This type corresponds to the group of $2 n \times 2 n$ symplectic matrices and the combinatorial description for its supercharacter theory resembles the one for type $D$. In this case $C_{2 n}(q)$-partitions are defined as in 3.1 but we also allow $\operatorname{arcs} i \stackrel{a}{\sim}-i$. Similar arguments can be used in this case, producing a similar definition for product and coproduct over the graded vector space $\mathbf{S C}^{C}$ endowing it with a Hopf algebra structure.

We remind that this has been done only for $\operatorname{char}\left(\mathbb{F}_{q}\right) \geq 3$. The case $q=2$ requires a different description of the elements of the group $U_{2 n}^{D}(q)$. We want to understand this case as well, since this might allow us to have an unlabelled version of what we have done here. This is part of forthcoming work by the author. However, a coarser version of this supercharacter theory could have some connection with this case. Namely, by lumping together conjugacy classes in $U_{2 n}^{D}(q)$ through the action $\left(B_{2 n}(q) A B_{2 n}(q)+I_{2 n}\right) \cap U_{2 n}^{D}(q)$, where $B_{2 n}(q)$ is the Borel subgroup of $G L_{2 n}(q), A \in \mathfrak{u}^{D}(q)$, gives a coarser superclass theory which does not depend on $q$ (though the supercharacters do). Hence, the Hopf algebra constructed here would realize the unlabelled version given by this super-theory.

Finally, we want to point out that types $C$ and $D$ not only have a Hopf algebra structure, a Hopf monoid structure can be provided too. This is done in analogy to [ABT11].

\section{Acknowledgements}

I would like to express my gratitude to my supervisor Nantel Bergeron for introducing me into the world of supercharacters and Hopf algebras. Also, I thank to Carlos André and Nat Thiem for fruitful conversations.

\section{References}

$\left[\mathrm{AAB}^{+} 11\right]$ M. Aguiar, C. André, C. Benedetti, N. Bergeron, Z. Chen, P. Diaconis, A. Hendrickson, S. Hsiao, M. Isaacs, A. Jedwab, K. Johnson, G. Karaali, A. Lauve, T. Le, S. Lewis, H. Li, K. Magaard, E. Marberg, J-C. Novelli, A. Pang, F. Saliola, L. Tevlin, J-Y. Thibon, N. Thiem, V. Venkateswaran, C. Vinroot, N. Yan, and M. Zabrocki. Supercharacters, symmetric functions in noncommuting variables. In DMTCS Proc. AO, 2011.

[ABT11] M. Aguiar, N. Bergeron, and N. Thiem. A Hopf monoid from the representation theory of the finite group of unitriangular matrices. In preparation, October 2011.

[AL11] M. Aguiar and A. Lauve. Lagrange's theorem for hopf monoids in species. In DMTCS Proc. AO, 2011. 
[AN09a] C. André and A. Neto. A supercharacter theory for the sylow $p$-subgroups of the finite symplectic and orthogonal groups. J Algebra., 322:1273-1294, 2009.

[AN09b] C. André and A. Neto. Supercharacters of the sylow $p$-subgroups of the finite symplectic and orthogonal groups. Pacific Journal of Math., 239(2):201-230, 2009.

[And95] C. André. Basic characters of the unitriangular group. J Algebra, 175(1):287-319, 1995.

[Bon68] Mars Bonfire. Born to be wild. From the album Steppenwolf, by Steppenwolf, January 1968.

[BS03] G. Belitskii and V. Sergeichuk. Complexity of matrix problems. Linear algebra appl.., 361:203-222, 2003.

[BZ09] N. Bergeron and M. Zabrocki. The hopf algebras of symmetric functions and quasi-symmetric functions in non-commutative variables are free and cofree. J Algebra Appl, 8(4):581-600, 2009.

[Car72] R.W. Carter. Simple groups of Lie type. Pure App. Math., 1972.

[DI08] P. Diaconis and M. Isaacs. Supercharacters and superclasses for algebra groups. Trans. Amer. Math. Soc., 360(5):2359-2392, 2008.

[Sag01] B. Sagan. The symmetric group. Representations, Combinatorial algorithms and symmetric functions. Springer, 2nd edition, 2001.

[Thi10] N. Thiem. Branching rules in the ring of superclass functions of unipotent upper-triangular matrices. J Algebraic Combin., 31(2):267-298, 2010. 